University of Nebraska - Lincoln

DigitalCommons@University of Nebraska - Lincoln

Faculty Publications from the Department of

Electrical and Computer Engineering

\title{
Broadband plasmonic-enhanced forward and backward multiplex coherent anti-Stokes Raman scattering microscopy
}

Baoshan Guo

Lan Jiang

Yanhong Hua

Xin Li

Tianhong Cui

See next page for additional authors

Follow this and additional works at: https://digitalcommons.unl.edu/electricalengineeringfacpub

Part of the Computer Engineering Commons, and the Electrical and Computer Engineering Commons

This Article is brought to you for free and open access by the Electrical \& Computer Engineering, Department of at DigitalCommons@University of Nebraska - Lincoln. It has been accepted for inclusion in Faculty Publications from the Department of Electrical and Computer Engineering by an authorized administrator of DigitalCommons@University of Nebraska - Lincoln. 
Authors

Baoshan Guo, Lan Jiang, Yanhong Hua, Xin Li, Tianhong Cui, and Yongfeng Lu 


\title{
Optical Engineering
}

\section{Broadband plasmonic-enhanced forward and backward multiplex coherent anti-Stokes Raman scattering microscopy}

\author{
Baoshan Guo \\ Lan Jiang \\ Yanhong Hua \\ Xin Li \\ Tianhong Cui \\ Yongfeng Lu
}




\title{
Broadband plasmonic-enhanced forward and backward multiplex coherent anti-Stokes Raman scattering microscopy
}

\author{
Baoshan Guo,, Lan Jiang, ${ }^{a, *}$ Yanhong Hua, ${ }^{a}$ Xin Li, ${ }^{a}$ Tianhong Cui, ${ }^{b}$ and Yongfeng Lu ${ }^{c}$ \\ ${ }^{a}$ Beijing Institute of Technology, School of Mechanical Engineering, Laser Micro/Nano Fabrication Laboratory, Beijing, China \\ bUniversity of Minnesota, Department of Mechanical Engineering, Minneapolis, Minnesota, United States \\ 'University of Nebraska-Lincoln, Department of Electrical Engineering, Lincoln, Nebraska, United States
}

\begin{abstract}
Coherent anti-Stokes Raman scattering (CARS) microscopy is an attractive technique for label-free biochemical-specific characterization of biological specimens. However, it has very low sensitivity in monitoring and imaging molecules present in extremely low concentrations or at fast speeds. To improve this sensitivity, especially for multiplex CARS, the intensity of the pump beam and broadband Stokes beam should be enhanced simultaneously. Therefore, the gold shell particle and gold surface are demonstrated to enhance the forward and backward CARS, respectively. Results show that a signal enhancement factor of $\sim 25,000$ can be achieved for the gold surface and an even higher enhancement factor can be achieved for the gold shell particles. Thus, we can obtain an enhanced CARS signal in a broad spectral range, which will substantially improve the detection sensitivity of hyperspectral CARS spectroscopy and imaging. ๑ 2018 Society of Photo-Optical Instrumentation Engineers (SPIE) [DOI: 10.1117/1.OE.57.3.033105]
\end{abstract}

Keywords: plasmonics; coherent anti-Stokes Raman scattering; nanoparticles; core-shell particles.

Paper 180070 received Jan. 13, 2018; accepted for publication Mar. 6, 2018; published online Mar. 20, 2018.

\section{Introduction}

Coherent anti-Stokes Raman scattering (CARS), a nonlinear four-wave mixing process that has substantially higher sensitivity than does spontaneous Raman scattering, has been employed in vibrational bioimaging since the late 1990s. ${ }^{1}$ CARS is a third-order nonlinear effect where three photons $\omega_{1}, \omega_{2}$, and $\omega_{3}$ with two frequencies $\omega_{s}$ and $\omega_{p}$ interact coherently through the third-order susceptibility $\left[\chi^{(3)}\right]$ of the material, producing a spectrally separated and blueshifted photon at the anti-Stokes frequency $\omega_{\text {as }}$ (Fig. 1).

CARS microscopy has been an attractive technique for label-free biochemical-specific characterization of biological specimens. ${ }^{2}$ Compared with spontaneous Raman scattering, CARS offers signals that are stronger by several orders of magnitude, which enables sensitive chemoselective imaging at the video rate. ${ }^{3}$ Conventional instrumentation of CARS utilizes two synchronized lasers or optical parametric oscillators with a frequency difference to obtain a single-color or narrowband CARS image. ${ }^{4}$ However, the richness of the broadband spectral Raman information in the sample is not efficiently utilized in the aforementioned narrowband CARS excitation method. Therefore, multiplex CARS (MCARS) microscopy based on supercontinuum (SC) generation was developed; this technique yields broadband CARS spectra in an individual image pixel, and these spectra can indicate the intrinsic chemical information of the sample. ${ }^{5}$

Although CARS is more sensitive than conventional in Raman scattering due to its higher-order dependence on incident power, its sensitivity is currently insufficient for monitoring or imaging the molecules present in extremely low concentrations. Moreover, reduced photodamage and faster real-time acquisition are highly desirable in bioimaging applications. Hence, it is crucial for achieving higher sensitivities to meet these requirements. One approach to achieve higher sensitivities is to employ surface plasmons generated on nanostructures to enhance CARS signals. Although plasmonic enhancement of Raman scattering ${ }^{6-8}$ and fluorescence on surfaces ${ }^{9}$ has been widely studied over the last several decades, only a few such attempts have been made with CARS; a few studies have focused on colloidal silver and gold nanoparticles. ${ }^{10-14}$ The colloidal silver and gold nanoparticles used in the forward CARS only work at a single wavelength, which is unsuitable for broadband M-CARS. Yampolsky et al. reported using a type of gold nanodumbbells encapsulated in porous silica shells to enhance the CARS signal. ${ }^{15}$ However, the enhancing effect only works at the junction between two gold nanospheres, and this effect is a narrowband effect similar to that of a regular gold particle. Furthermore, Hayazawa et al. reported the enhancement of the CARS signal using modified atomic force microscopy tips (a process termed tip-enhanced CARS) ${ }^{16,17}$ However, for forward CARS, finding an appropriate structure that can enhance the signal in a broad spectrum remains difficult. In addition, the type of gold surface used in Ref. 18 can be used for backward CARS (also called epi-CARS), a process that can yield some chemical information that conventional forward CARS signals cannot. However, fabricating this structure is complicated, and the sharp tip can get easily burned by the largely enhanced optical field.

In this study, a type of gold shell particle having a broadband plasmonic-enhancing spectrum is demonstrated theoretically. The pump, Stokes, and broadband anti-Stokes 


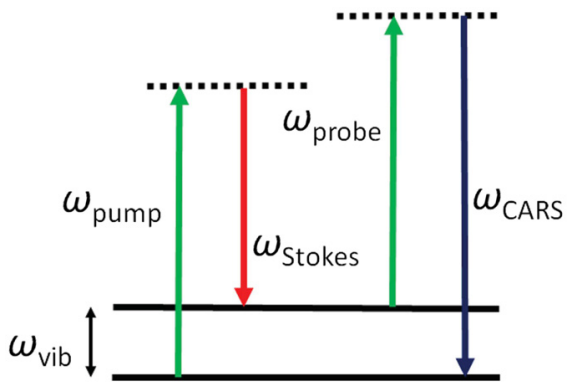

Fig. 1 Schematic band energy diagram of CARS.

photons can simultaneously be in resonance with the particle, a phenomenon impossible to realize with simple solid gold particles. Furthermore, the resonances can be easily tuned by varying the core size, core materials, and shell thickness of these particles to match any suitable laser. By using the broadband plasmonic-enhancing effect, this type of particle can enhance the M-CARS signal in a broad spectral range, which in turn largely improves the detection sensitivity of forward hyperspectral CARS imaging. In addition to simulating the plasmonic-enhanced forward M-CARS, we experimentally demonstrated a type of simple gold surface to enhance the backward M-CARS signal.

\section{Experimental Setup}

Figure 2 shows the experimental setup of our M-CARS system. A high-power Ti:Sapphire mode-locked laser (Mai Tai BB, Newport) was used to provide the pump and Stokes for CARS. The laser output pulse had a center wavelength of $800 \mathrm{~nm}$ with a pulse width of $80 \mathrm{fs}(80 \mathrm{MHz}$ repetition rate). After passing through a Faraday isolator, the pulse width of the signal considerably broadened; therefore, it was compressed back to a transform-limited pulse using a prism-pair compressor. The power ratio after using the polarization beam splitter can be optimized by tuning the polarization of the laser with a half-wave plate. We split the $\sim 100$-mW laser power for coupling into a photonic-crystal fiber (PCF) (femtoWHITE-800, NKT) as the pump for $\mathrm{SC}$ generation, pumped at the anomalous dispersion regime. The output power of the PCF was $\sim 22 \mathrm{~mW}$, which resulted in a broadband SC covering a Raman shift range greater than $3000 \mathrm{~cm}^{-1}$. Thereafter, we selected the output signal with a wavelength larger than $805 \mathrm{~nm}$ as the Stokes beam by employing a long-pass filter. Because the bandwidth of
Ti:Sapphire laser was $\sim 10 \mathrm{~nm}$, we used a 4- $f$ grating pair with a slit to narrow the bandwidth of the pump, thus improving the spectral resolution for M-CARS spectra. After the generation of Stokes and the spectral narrowing of the pump, we combined the two beams using a dichroic filter. The temporal overlap between the two beams can be adjusted by tuning the delay line in the Stokes path. An objective lens with a high numerical aperture $(\mathrm{NA}=0.85)$ was used to focus the two beams on the sample. This tight focusing condition can relax the phase-matching condition for the generation of the anti-Stokes signal. Moreover, it can reduce the background signal from the substrate and provide a high diffraction-limited resolution. The power of the pump beam and stokes beam focused on the sample was $\sim 8$ and $5 \mathrm{~mW}$, respectively. Similar to a conventional microscope, the objective lens of our system is tunable. After passing through the collimating objective, the CARS signal was separated using a short-pass filter, which blocked the pump and Stokes beam. Consequently, the filtered anti-Stokes had a broad spectral range of $\sim 2000 \mathrm{~cm}^{-1}(\sim 600$ to $780 \mathrm{~nm})$. The M-CARS signal thus covers most of the characteristic vibrational frequencies for biological samples. We used a spectrometer (Acton SP2300, Princeton Instruments) with a sensitive cooled charge-coupled device (PIXIS 100, Princeton Instruments) to detect the CARS signal.

\section{Experiment and Simulation Results}

The proposed system was used to obtain the CARS signal of dimethyl sulfoxide (DMSO) [Fig. 3(a)]. Given the differences in the delay time of different wavelengths of the broad Stokes pulse, the intensity of different signal peaks can be relatively changed by tuning the delay time between the pump and Stokes pulses. The time delay was fixed to achieve a large clear DMSO signal peak at $670 \mathrm{~cm}^{-1}$. Subsequently, the gold nanoparticles were added to the DMSO and mixed to enhance the CARS signal. The gold particles detected using a transmission electron microscope are shown in Fig. 3(b). The gold particles in the solution were $\sim 50 \mathrm{~nm}$ in diameter.

Figure 3(a) shows the DMSO CARS signals detected with (black square-dotted line) and without (red circle-dotted line) the gold particles. The peak intensity at $670 \mathrm{~cm}^{-1}$ was enhanced more than 10 -fold by the gold particles. However, in the large wavenumber region, similar to the peak at $2900 \mathrm{~cm}^{-1}$, the signal intensity was not enhanced, confirming that the enhancing effect was only a narrowband

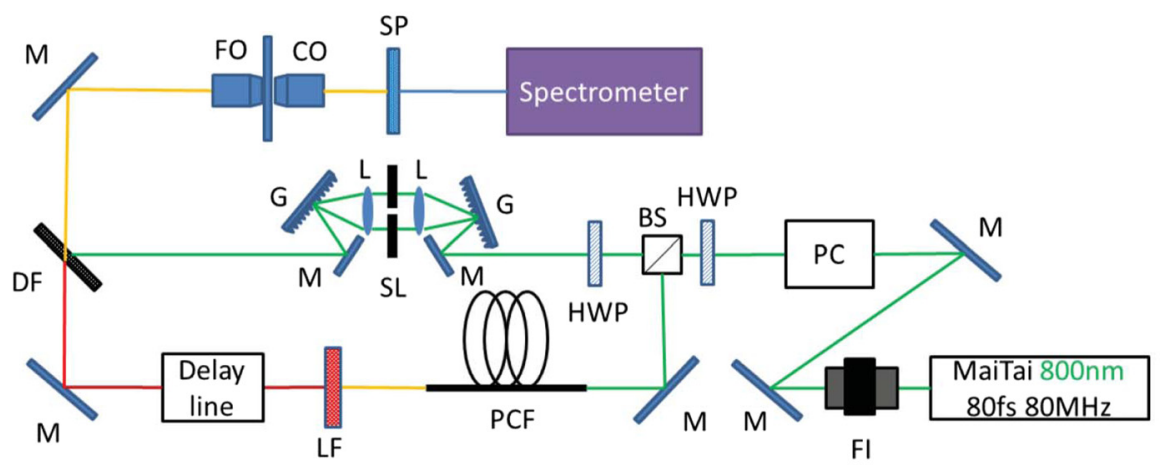

Fig. 2 Schematic of the M-CARS system. FI, Faraday isolator; M, mirror; PC, prism compressor; HWP, half-wave plate; BS, beam splitter; PCF, photonic crystal fiber; LF, long-pass filter; G, grating; L, lens; $\mathrm{SL}$, slit; DF, dichroic filter; FO, focus objective; CO, collimating objective; SP, short-pass filter. 


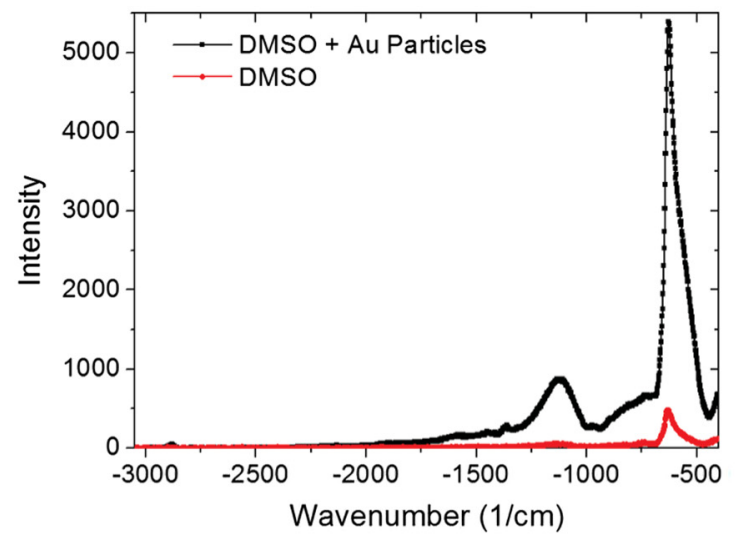

(a)

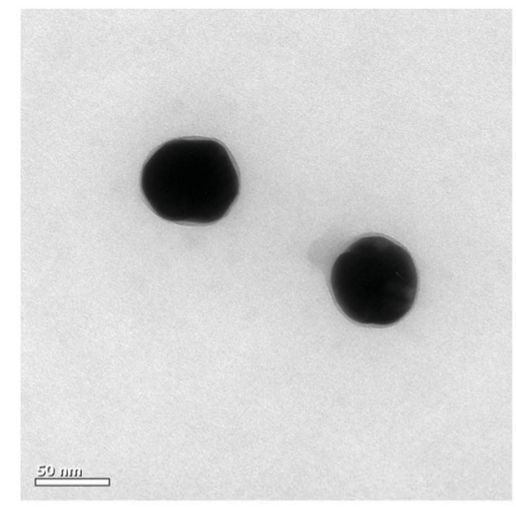

(b)

Fig. 3 (a) Enhanced gold particles and normal CARS signal of DMSO and (b) TEM image of gold particles.

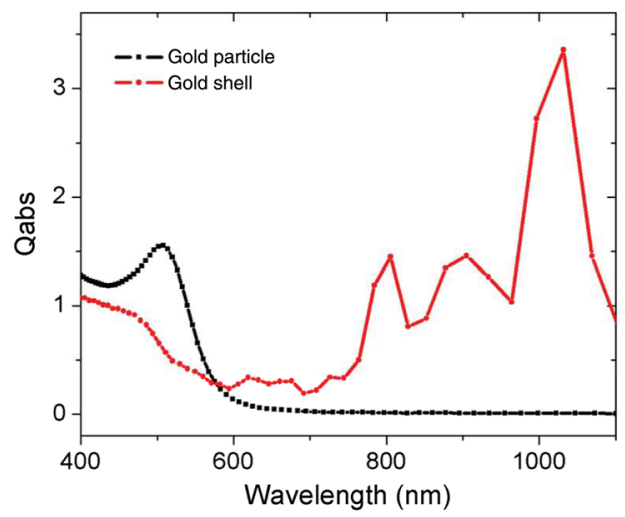

Fig. 4 Plasmonic enhancement absorption spectra of gold particle (black dotted curve) and gold shell particle (red solid curve).

effect, which is unfavorable for M-CARS. Figure 4 presents the theoretical results for gold particle-induced enhancement, calculated using the finite-difference time-domain (FDTD) method. The resonant spectrum is narrow and only has an enhanced absorption peak at around $526 \mathrm{~nm}$. The enhancing factor drastically decreases in the other wavelength region (black dotted curve in Fig. 4). In particular, in the longer wavelength region larger than $600 \mathrm{~nm}$, the enhancing factor is nearly zero, indicating that the solid gold sphere particles had only a little effect on enhancement of the pump beam $(800 \mathrm{~nm})$ and Stokes beam $(805$ to $1100 \mathrm{~nm})$. The signal enhancement in the experiment can be attributed to the nonconformity or special shape of the real particles [Fig. 3(b)]. When gold shell particles were used, a very broad enhancing spectrum was obtained (red solid curve in Fig. 4). The enhancing effect, especially in the 750 to $1100 \mathrm{~nm}$ region, was considerably larger than that for the simple gold particles. In our experimental system, the pump wavelength was $800 \mathrm{~nm}$, and the broadband Stokes beam covered the region from 805 to $1100 \mathrm{~nm}$. Therefore, the pump beam and Stokes beam were all located in the enhanced spectral region. The intensity of conventional CARS signals can be attributed to third-order nonlinear polarization; this intensity is dependent on the square of the pump and is linearly dependent on the Stokes field intensity ${ }^{2}$ : $I_{\text {CARS }} \propto I_{P}^{2} I_{S}$. Therefore, the simultaneous enhancement of pump beam and Stokes beam is crucial and substantially enhances the CARS signal.

The gold shell particle has a gold shell of thickness $20 \mathrm{~nm}$ and a silica core of diameter $140 \mathrm{~nm}$. Figure 5 shows the optical field distribution of the gold particle and gold shell particles simulated using the FDTD method. The optical field distribution of gold particles in various cross sections is shown in Figs. 5(a)-5(c). The highest resonant peak of the gold particles was $526.17 \mathrm{~nm}$, and the intensity was enhanced $\sim 28$-fold [Figs. 5(b) and 5(c)]. For the gold shell particles [Figs. 5(d)-5(f)], the highest resonant peak was at $1019.35 \mathrm{~nm}$, with 120-fold enhancement in intensity. Notably, rather than a single peak, enhanced broadband peaks were observed for the gold shell particles (Fig. 4). Accordingly, we conclude that the CARS intensity and detection sensitivity can be considerably improved using gold shell particles. The resonant peak of the gold shell particles can be tuned by changing the core size, core materials, and shell thickness. Therefore, gold shell particles are suitable for enhancing the detection sensitivity of the forward M-CARS system in different spectral ranges.

In addition to simulating enhanced broadband forward M-CARS, we experimentally investigated a gold surface (Fig. 6) as a medium to enhance the backward M-CARS (epi-M-CARS) signal. The epi-CARS signal contains different information than does the forward CARS signal. In general, the epi-CARS signal appears in positions where the forward CARS signal is absent. ${ }^{19}$ The used gold surface is not transparent as all of the incoming light is either reflected or absorbed. The gold surface (film) was deposited on a glass substrate of thickness $\sim 100 \mathrm{~nm}$. Periodic holes of diameter $\sim 240 \mathrm{~nm}$ were etched at intervals of $780 \mathrm{~nm}$. Figure 6 shows the scanning electron microscopy image of such a gold surface.

The DMSO sample was placed on a glass slide. A normal, flat gold surface and the structured gold surface were used to cover the DMSO sample to generate the conventional backward M-CARS signal and surface-enhanced backward CARS signal, respectively. Figure 7(a) presents the detected DMSO signal. The flat gold surface reflects most of the incoming light. Hence, the intensity of the epi-M-CARS signal was lower than that of the forward CARS signal (red curve in Fig. 3). The peak intensity of the epi-M-CARS 


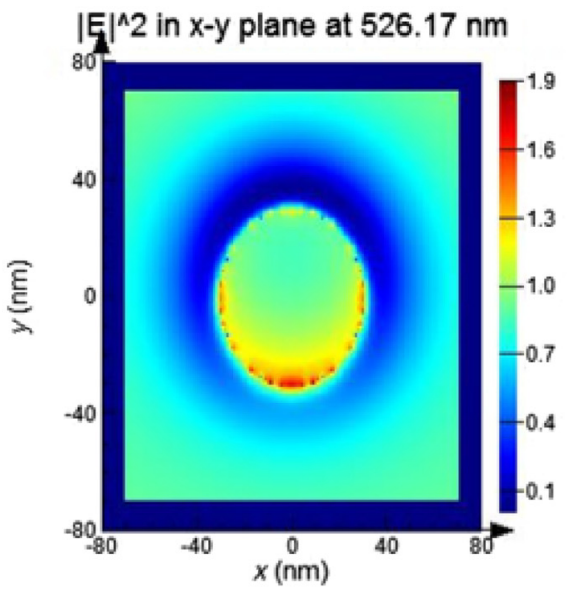

(a)

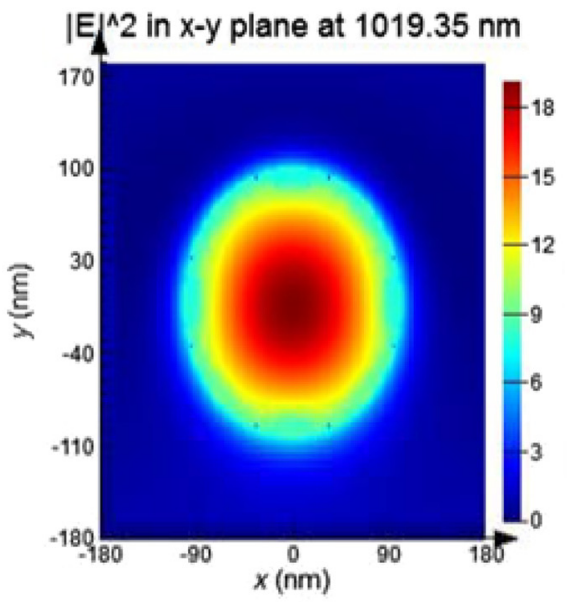

(d)

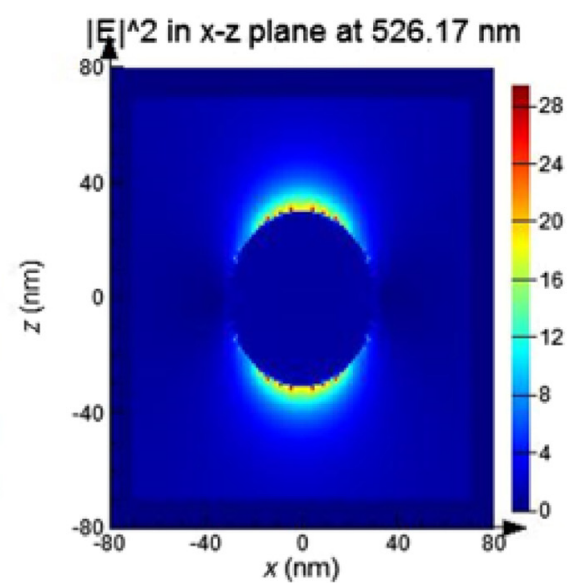

(b)

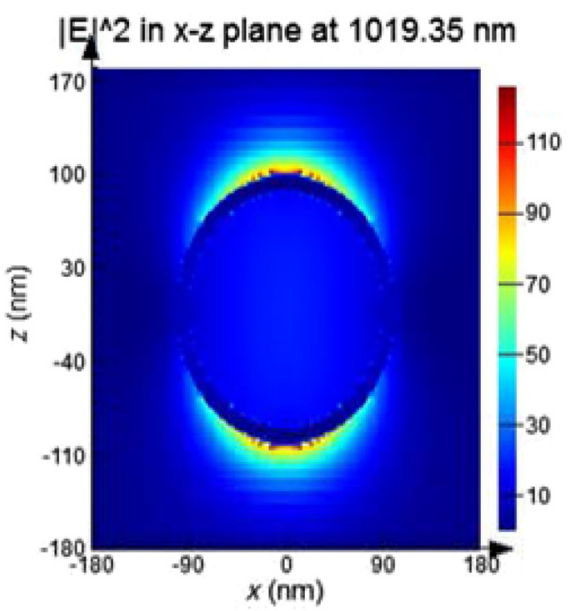

(e)

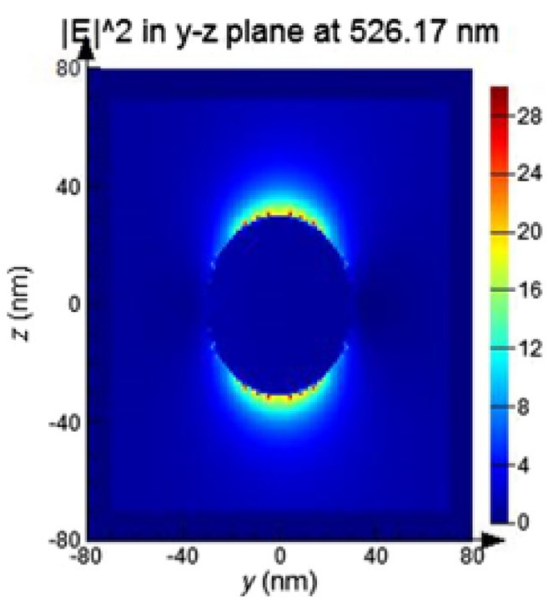

(c)

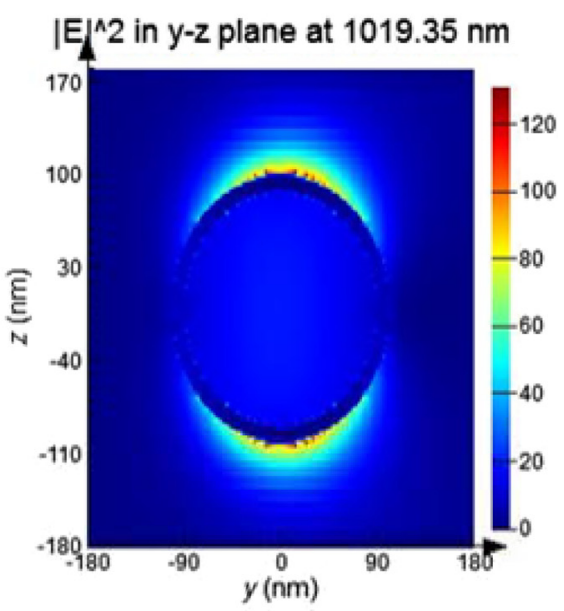

(f)

Fig. 5 Resonant peaks of the gold particles [top line (a), (b), (c)] and gold shell particles [bottom line (d), (e), (f)] at various cross sections. The polarization of light field is in the $Z$ direction.

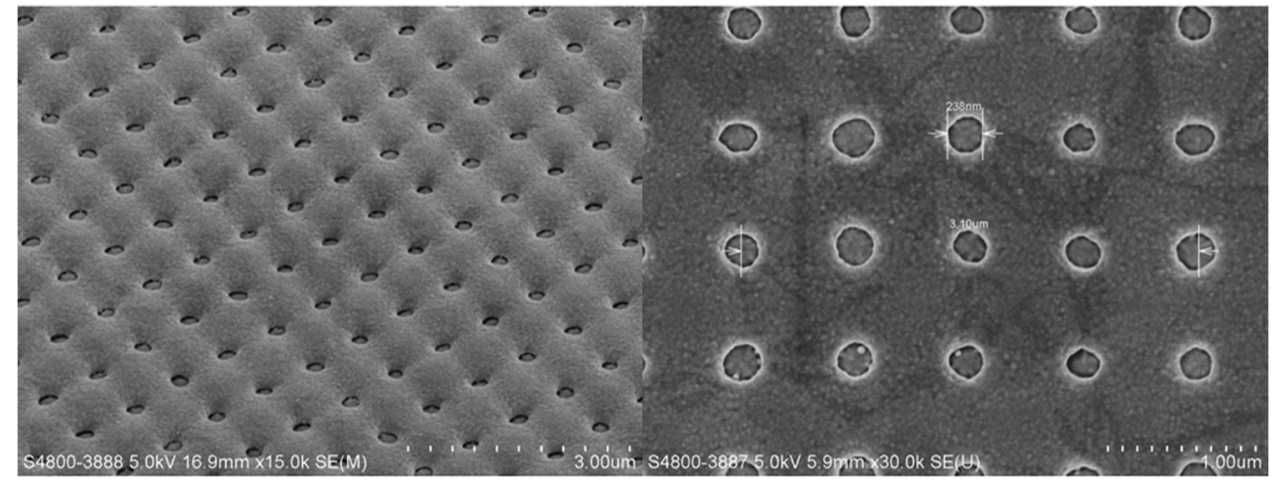

(a)

(b)

Fig. 6 (a) Scanning electron microscopy image of a gold film of thickness $100 \mathrm{~nm}$, (b) with periodic holes of diameter $\sim 240 \mathrm{~nm}$ etched at intervals of $780 \mathrm{~nm}$.

signal at $670 \mathrm{~cm}^{-1}$ was enhanced by at least 25 -fold when using the structured gold surface [black curve in Fig. 7(a)], which had a considerably higher enhancing factor than that in the experiment with solid gold particles. Notably, in the large wavenumber region, the signal intensity of the peak at $2900 \mathrm{~cm}^{-1}$ was substantially enhanced, which is attributable to the considerably higher pump beam enhancement by the gold surface. Figure 7(b) shows the simulated reflection spectrum of the surface. The peak enhancing wavelength was $800 \mathrm{~nm}$, which is in agreement with the pump beam wavelength. The gold surface enhancing the optical field distribution at $800 \mathrm{~nm}$ is shown in Fig. 7(c). The optical field is polarized in the $X$ direction, so the plasmonic-enhancing effect mainly occurs in this direction. Hence, the enhancing 


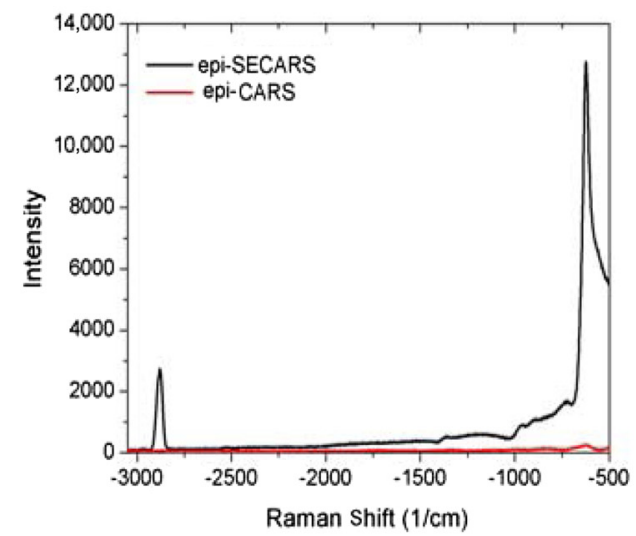

(a)

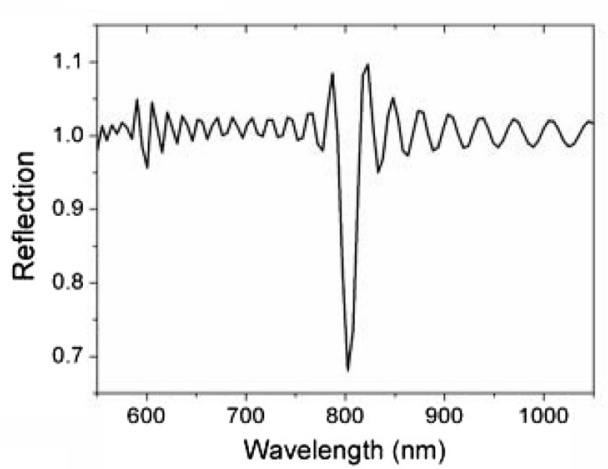

(b)

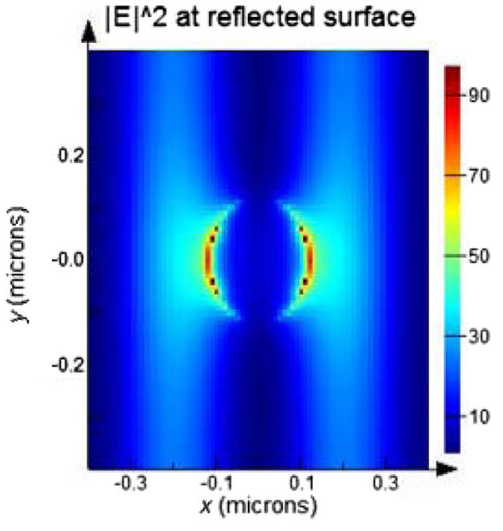

(c)

Fig. 7 (a) Surface-enhanced epi-CARS (black line) and normal transmitted CARS signal (red line) of DMSO, (b) reflection spectrum of the gold surface, and (c) optical field distribution at the resonant wavelength of $800 \mathrm{~nm}$ on the reflected surface.

optical field around the surface hole shows an anisotropic pattern. This type of gold surface is simple and easy to fabricate and is a stable method of obtaining a plasmonicenhanced epi-M-CARS signal. The maximum enhancement intensity for the gold surface sample was $\sim 90$-fold [Fig. 7(c)], which is higher than that for the solid gold particles but lower than that for the gold shell particles.

Thus, the gold shell particles have the strongest enhancement effect and are therefore suitable for forward M-CARS. However, the precise fabrication of gold shell particles is challenging. If this problem can be overcome, a considerable improvement in the high-sensitivity M-CARS system can be realized. Furthermore, we can use a concept similar to the gold shell structure to change the gold surface, such as changing the glass substrate to silicon substrate, etching a hole array, and grating on the silicon substrate followed by gold-film deposition. Per the simulation, such a surface with a hole array or a grating has a much wider enhanced absorption spectrum, and the enhanced peak is also much higher. In addition, gold shell particles can be used together with a gold surface to further enhance epi-M-CARS. Therefore, the detection sensitivity of epi-M-CARS can be improved substantially using both gold shell particles and a gold surface, which will be helpful for realizing a highspeed CARS system. ${ }^{20}$

The number of molecules in the focus and surface can be calculated in either case. ${ }^{18}$ Subsequently, a molecular enhancement factor $G$ can be calculated, similar to the SERS substrate enhancement factor.

First, the focal volume can be calculated as

$V_{\text {Focus }}=\pi \times\left(\frac{D_{\text {spot }}}{2}\right)^{2} \times\left(1.5 \times D_{\text {spot }}\right)$.

The number of molecules in the focal volume can be calculated as

$N_{\mathrm{vol}}=\frac{\rho}{M} \times \mathrm{NA} \times V_{\text {Focus }}$.

The number of molecules in the focal surface can be calculated as
$N_{\text {surf }}=\frac{\rho}{M} \times \mathrm{NA} \div 10^{7} \times \pi \times\left(\frac{D_{\text {spot }}}{2}\right)^{2}$

The enhancement factor can be calculated as

$$
\begin{aligned}
G_{\text {SECARS }} & =\frac{\frac{I_{\text {SECARS }}}{N_{\text {surf }}}}{\frac{I_{\text {CARS }}}{N_{\text {vol }}}}=\frac{I_{\text {SECARS }} \times N_{\text {vol }}}{I_{\text {CARS }} \times N_{\text {surf }}} \\
& =\frac{I_{\text {SECARS }} \times \frac{\rho}{M} \times \mathrm{NA} \times \pi\left(\frac{D_{\text {spot }}}{2}\right)^{2}\left(1.5 D_{\text {spot }}\right)}{I_{\text {CARS }} \times \frac{\rho}{M} \times \mathrm{NA} \div 10^{7} \times \pi\left(\frac{D_{\text {spot }}}{2}\right)^{2}} \\
& =\frac{I_{\text {SECARS }} \times\left[1.5 D_{\text {spot }}(\mathrm{cm})\right]}{I_{\text {CARS }} \div 10^{7}} \\
& =\frac{I_{\text {SECARS }} \times 1.5 D_{\text {spot }}(\mathrm{nm})}{I_{\mathrm{CARS}}} .
\end{aligned}
$$

In our case, the diameter of the focal spot, $D_{\text {spot }}=0.61 \lambda / \mathrm{NA}, \lambda=800 \mathrm{~nm}, \quad \mathrm{NA}=0.85$, and thus, $D_{\text {spot }} \approx 574 \mathrm{~nm}$. Figure 7 (a) shows the peak intensity at $670 \mathrm{~cm}^{-1}: I_{\text {SECARS }} \approx 13,000, I_{\text {CARS }} \approx 300$. The molecular enhancement factor $G_{\text {SECARS }}$ was $\sim 25,000$. Importantly, the enhancement was not restricted to only one signal peak.

\section{Conclusion}

Plasmonic nanoparticles and surface considerably enhanced the signal of the M-CARS system. A gold shell particle was numerically demonstrated to simultaneously enhance the pump and broadband Stokes beam to realize a broadband plasmonic-enhancing M-CARS system. The forward and backward M-CARS system can be applied to detect transparent and opaque samples, respectively. The detection sensitivity of the M-CARS spectrum and hyperspectral imaging can be improved considerably using broadband-enhanced plasmonic structures; this is especially important for low-concentration detection and high-speed CARS systems. Thus, different molecules can be detected and recorded simultaneously in a broad spectrum using the M-CARS system. For the application in biological samples, one potential problem is the heat dissipation from the gold particles because of the low biological damage threshold. The gold particles or 
gold surface can be destroyed under high laser power. In fact, we must always be careful about the input laser power for the plasmonic enhancing or any other optical signal detection. In general, this study paves the way for reliable molecule spectroscopy as well as high-speed, sensitive molecular imaging with plasmonic-enhanced M-CARS.

\section{Acknowledgments}

This study was supported by the National Key R\&D Program of China (2017YFB1104300) and the National Natural Science Foundation of China (NSFC) under grant no. 61605140 .

\section{References}

1. A. Zumbusch, G. R. Holtom, and X. S. Xie, "Three-dimensional vibrational imaging by coherent anti-Stokes Raman scattering," Phys. Rev. Lett. 82, 4142-4145 (1999).

2. C. L. Evans and X. S. Xie, "Coherent anti-Stokes Raman scattering microscopy: chemical imaging for biology and medicine," Аnпu. Rev. Anal. Chem. 1, 883-909 (2008).

3. M. Fleischmann, P. Hendra, and A. McQuillan, "Raman spectra of pyridine adsorbed at a silver electrode," Chem. Phys. Lett. 26, 163-166 (1974).

4. C. L. Evans et al., "Chemical imaging of tissue in vivo with video-rate coherent anti-Stokes Raman scattering microscopy," Proc. Natl. Acad. Sci. U. S. A. 102(46), 16807-16812 (2005).

5. E. O. Potma et al., "High-sensitivity coherent anti-Stokes Raman scattering microscopy with two tightly synchronized picosecond lasers," Opt. Lett. 27(13), 1168-1170 (2002).

6. H. N. Paulsen et al., "Coherent anti-Stokes Raman scattering microscopy with a photonic crystal fiber based light source," Opt. Lett. 28(13), 1123-1125 (2003).
7. D. L. Jeanmaire and R. P. Van Duyne, "Surface Raman spectroelectrochemistry. 1. Heterocyclic, aromatic, and aliphatic-amines adsorbed on anodized silver electrode," J. Electroanal. Chem. 84, 1-20 (1977).

8. K. Kneipp, M. Moskovits, and H. Kneipp, Surface-Enhanced Raman Scattering: Physics and Applications, Springer, New York (2006).

9. J. R. Lakowicz, "Radiative decay engineering: biophysical and biomedical applications," Anal. Biochem. 298, 1-24 (2001).

10. T. Koo, S. Chan, and A. A. Berlin, "Single-molecule detection of biomolecules by surface-enhanced coherent anti-Stokes Raman scattering," Opt. Lett. 30, 1024-1026 (2005).

11. E. Liang et al. "Experimental observation of surface-enhanced coherent anti-Stokes Raman scattering," Chem. Phys. Lett. 227, 115-120 (1994).

12. V. Namboodiri et al., "Surface-enhanced femtosecond CARS spectroscopy (SE-CARS) on pyridine," Vib. Spectrosc. 56, 9-12 (2011).

13. C. J. Addison et al., "Tuning gold nanoparticle self-assembly for optimum coherent anti-Stokes Raman scattering and second harmonic generation response," J. Phys. Chem. C 113, 3586-3592 (2009).

14. T. Ichimura et al., "Local enhancement of coherent anti-Stokes Raman scattering by isolated gold nanoparticles," J. Raman Spectrosc. 34, 651654 (2003)

15. S. Yampolsky et al., "Seeing a single molecule vibrate through timeresolved coherent anti-Stokes Raman scattering," Nat. Photonics 8, 650-656 (2014).

16. N. Hayazawa et al., "Amplification of coherent anti-Stokes Raman scattering by a metallic nanostructure for a high resolution vibration microscopy," J. Appl. Phys. 95, 2676-2681 (2004).

17. T. Ichimura et al., "Tip-enhanced coherent anti-Stokes Raman scattering for vibrational nano-imaging," Phys. Rev. Lett. 92, 20-23 (2004).

18. C. Steuwe et al., "Surface enhanced coherent anti-Stokes Raman scattering on nanostructured gold surfaces," Nano Lett. 11, 5339-5343 (2011).

19. I. W. Schie et al., "Simultaneous forward and epi-CARS microscopy with a single detector by time correlated single photon counting," Opt. Express 15, 2168-2175 (2008).

20. K. Hashimoto et al., "Broadband coherent Raman spectroscopy running at 24, 000 spectra per second," Sci. Rep. 6, 21036 (2016).

Biographies for the authors are not available. 